\title{
The Blood Biomarkers of Thyroid Cancer
}

This article was published in the following Dove Press journal:

Cancer Management and Research

\section{Weiran Wang (ID \\ Jingtao Chang \\ Baosong Jia \\ Jing Liu}

First Hospital of Shanxi Medical University, General Surgery Department, Taiyuan, Shanxi, People's Republic of China
Correspondence: Jing Liu Email liujing5585@I63.com
Introduction: With the gradual increase in the incidence of thyroid cancer, people's attention to thyroid cancer has also gradually increased. Although the prognosis of thyroid cancer is rather mild compared to other cancers, it will still bring a heavy psychological burden on people who have been diagnosed. At present, the diagnosis of thyroid cancer mainly depends on ultrasound and percutaneous fine needle aspiration (pFNA). Due to the unsatisfactory accuracy of the diagnosis methods we use now, there are still some thyroid nodules that cannot be clearly diagnosed before surgery.

Methods: In this article, we have searched for relevant research on blood markers of thyroid cancer in the past five years and categoried them into four groups.

Discussion: Though we have not found a biomarker which can diagnose thyroid cancer both sensitively and specifically, we do found many substances that are related to it, and have the potential to recognize it and help the diagnosis. And perhaps combined models can do it better.

Keywords: thyroid neoplasms, blood biomarkers, diagnosis

\section{Introduction}

In the past ten years, the incidence of thyroid cancer has been increasing yearly, and it has become the fourth highest in women; ${ }^{1}$ Part of the reason might due to the rapid development of imaging detection technologies and continuously increasing awareness of people's health. But at the same time, data show that the incidence of advanced thyroid cancer and the diagnosis of low-risk thyroid cancer are also rising, so this phenomenon cannot be explained only by overdiagnosis. ${ }^{2}$ Current initial diagnostic methods mainly rely on ultrasound, and the gold standard for screening benign and malignant thyroid nodules is percutaneous fine needle puncture biopsy (pFNA) or intraoperative frozen pathological results. ${ }^{3}$ But in view of the limited accuracy of ultrasound diagnosis, and the defect that pFNA depends too much on the diagnostic level of the pathology department of the medical institution and due to the small sample size, some specimens cannot be diagnosed, repeated puncture or intraoperative frozen pathology should be done to make the diagnosis, we need to find a biomarker to assist or even replace existing diagnostic methods. ${ }^{4}$

Blood is the most convenient and difficult-to-contaminate body fluid in the diagnosis of diseases, and various tumor markers in blood have been widely used in the diagnostic procedures, which confirmed its value in the diagnosis of tumors. As an important endocrine organ in the body, the thyroid has a wide range of effects on the human body, and its canceration will undoubtedly be reflected in the blood.

Biomarkers for thyroid cancer have been studied for more than 50 years. $^{5-7}$ Many people have made in this regard with a lot of results. This article reviews the 
results of tumor markers in the blood of thyroid cancer patients by categories in order to help find potential blood markers for thyroid cancer.

\section{Testing Methods and Means}

The determination of substances in blood is often combined with a variety of substance separation and analysis methods, such as nuclear magnetic resonance spectroscopy (MRI), mass spectrometry (MS), gas chromatography (GC), liquid chromatography (LC) and photoacoustic imaging etc. Through the combination of multiple technologies, the possible content that changes a lot in blood can be separated. Besides, for some markers, such as melatonin, they are mainly identified by enzyme-linked immunosorbent assay (ELISA).

\section{Blood Markers}

\section{Markers That Related to Metabolism}

As an organ participates in energy metabolism, the thyroid gland plays an important role in it. At the same time, tumor cells also show their unique metabolic characteristics in the human body, mainly as follows: cancer cells prefers to use glycolysis rather than aerobic cycle even in an aerobic environment, namely the Warburg effect; ${ }^{8}$ The main pathways involved in human metabolism include energy metabolism (glucose metabolism, lipid metabolism and TCA), protein transcription and synthesis, and synthesis of nucleic acids and phosphatidylcholines.

In a study by Wojtowicz et al, the serum and urine samples of healthy people and patients with thyroid disease were compared, and it was suggested that serum is more suitable as diagnostic material than urine. Compared with healthy people, the levels of valine, alanine, creatine, and tyrosine in the serum of patients with papillary thyroid carcinoma have decreased; compared with patients with benign nodules, the serum valine and lactic acid also have a significant decrease in content, while compared to patients with thyroid adenoma, only lactic acid levels decreases. ${ }^{9}$

Farrokhi Yekta et al utilized non-targeted 1H-NMR to detect the blood of 17 patients with multiple goiter, 17 with thyroid papillary cancer and 20 healthy volunteers. Compared to healthy volunteers, there are quite obvious variation in the level of myo-inositol, shark-inositol, tryptophan, alanine, lactic acid, homocysteine, 3-methylglutaric acid, asparagine, and aspartate in serum samples from patients with PTC. The content of aspartic acid, choline, and acetamide has also changed significantly. Compared with patients with multiple goiter, changes in citric acid, acetylcarnitine, glutamine, homoserine, glutathione, kynurenine, niacin, hippuric acid, tyrosine, tryptophan, $\beta$ alanine, and xanthine were more pronounced. ${ }^{10}$

Huang et al performed a metabonomic analysis of thyroid nodules from 1540 serum-plasma matches and 114 tissue samples. In this analysis, Huang et al found that there is a significant difference in metabolic phenotypes in blood between healthy people and patients with thyroid nodules. The metabolic disordered pathways identified from serum include glycerophospholipid metabolism, arachidonic acid metabolism, linoleic acid metabolism, alanine, aspartic and glutamic acid metabolism and D-glutamine metabolism. Different metabolic pathways identified from plasma include alanine, aspartic acid, and glutamic acid metabolism, aminoacyl-tRNA biosynthesis, cysteine and methionine metabolism, and taurine-subtaurine metabolism and selenium amino acid metabolism. However, in Huang's study, the metabolic profiles between benign nodules and PTC overlapped completely. Therefore, non-invasive blood tests based on metabolomics are not suitable for their differential diagnosis. ${ }^{11}$

But Wojakowska's and Guo's research has yielded different results. Wojakowska's ${ }^{12}$ research using tissues as the material, reached similar conclusions as Guo's ${ }^{13}$ which used serum and tissue as the material. They both have confirmed the diagnostic significance of phosphatidylcholines (34:1) phosphatidic acids (36:3) and sphingomyelins (34:1). Phosphatidylcholines (34:1) can distinguish nodular patients from healthy people, while phosphatidic acids (36:3) and sphingomyelins (34:1) can distinguish benign and malignant nodules. Therefore, Guo et al believe that a tool combining the three may be able to distinguish benign and malignant thyroid nodules from healthy people.

Lee et al used nanoflow ultrahigh performance liquid chromatography-electrospray ionization-tandem mass spectrometry (nUHPLC-ESI-MS/MS) technology to analyze the lipid profiling that may occur in five types of cancer. Compared with healthy people, it is found that the lipid profiling of patients with thyroid cancer often shows a different trend from other cancers, and the increase in lysophosphatidylinositols (18:0 and 18:1) and lysophosphatidylethanolamine (18:1 and 18:2) might can be a sign of distinguishing thyroid cancer. ${ }^{14}$

At the same time, Lu's study based on proteomics also believes that people with papillary thyroid cancer (PTC) can be identified by establishing a model of peptide ions. 
$\mathrm{Lu}$ et al collected blood samples from 88 patients with PTC, 31 patients with benign nodules, and 49 healthy individuals. They found that a model based on 6 peptide ions can be used as a biomarker to identify PTC. At the same time, fibrinogen $\alpha$ and complement $\mathrm{C} 4 \mathrm{~A} / \mathrm{B}$ are considered potential markers for diagnosis of PTC. ${ }^{15}$

\section{Markers Related to Thyroid Function}

Thyroglobulin is a large glycoprotein found in thyroid cells and glia. However, because it is also derived from normal thyroid tissue and cells, it cannot be used as a basis for diagnosing thyroid cancer. However, after total thyroidectomy and ablation treatment, the level of serum thyroglobulin can be used for long-term monitoring and accessing the risk of recurrence. However, it should be noted that the presence of $\mathrm{TgAb}$ may interfere with the measurement of $\mathrm{Tg}$ during the detection process, resulting in erroneous results. ${ }^{16}$ Although thyroglobulin does not distinguish thyroid cancer from normal thyroid tissue, some studies ${ }^{17,18}$ have shown that preoperative detection of thyroglobulin may help predict the tumor burden and lymph node metastasis of patients. A study in South Korea ${ }^{19}$ believes that patients with preoperative thyroid globulin above $13.15 \mathrm{ng} / \mathrm{mL}$ can be considered to have ipsilateral cervical lymph node metastasis, while above $30.05 \mathrm{ng} / \mathrm{mL}$ may also have contralateral cervical lymph node metastasis, if higher than $62.9 \mathrm{ng} / \mathrm{mL}$ is at risk for distant metastases. Therefore, although thyroglobulin cannot be used as a marker for the diagnosis of thyroid cancer, it has a pivotal position in the management of thyroid cancer.

Calcitonin, a peptide hormone secreted by thyroid follicular cells, has been widely used in the diagnosis of medullary thyroid cancer (MTC) and postoperative follow-up, ${ }^{3}$ but its role in the diagnosis of all types of thyroid cancer has always existed disputes. In their study, Unluhizarci et al suggested that although the mechanism is not clear, in addition to MTC, other types of thyroid diseases, including methylene inflammation and Hashimoto's thyroiditis, may also elevate serum calcitonin. ${ }^{20}$ Although in 2009, someone $^{21}$ also claimed that follicular and papillary cancer cells can release substances that have paracrine stimulating effects on $\mathrm{C}$ cells, and ultimately increase serum calcitonin levels. However, related research is not sufficient, and its diagnostic significance is not clear.

There have been conflicting reports on the relationship between serum selenium, copper, and magnesium levels and thyroid cancer. In 2015, Shen et al conducted a meta- analysis on this issue and finally showed that there was a significant correlation between serum selenium, copper, and magnesium levels and thyroid cancer. ${ }^{22}$ Serum selenium concentration was negatively correlated with the stage of thyroid cancer. In addition, serum copper levels in patients with thyroid cancer were higher than those in healthy controls, and low levels of serum magnesium were associated with thyroid cancer. Although geography and detection methods may affect experimental results. ${ }^{23}$ Although most studies support a decrease in serum selenium levels in patients with thyroid cancer, and supplementation with selenium may reduce the risk of certain diseases, the decline is not specific, except for thyroid cancer, including infections, many diseases, including Parkinson's, can cause selenium levels to decline. ${ }^{24}$

The significance of vitamin $\mathrm{D}$ in the diagnosis and prognosis of thyroid cancer is also conflicted, although in 2016 and 2017, Choi $^{25}$ and Danilovic ${ }^{26}$ denied serum 25 $(\mathrm{OH}) \mathrm{D}_{3}$ 's role in determining the risk of thyroid cancer in their studies respectively. But in 2018, Zhang et al found that the content of $1,25(\mathrm{OH})_{2} \mathrm{D}_{3}$ in the serum of patients with PTC is significantly lower than that of healthy people, so maybe $1,25(\mathrm{OH})_{2} \mathrm{D}_{3}$ can become a potential diagnostic marker for PTC. ${ }^{27} \mathrm{Hu}^{\prime} \mathrm{s}^{28}$ and Zhao's ${ }^{29}$ further studies suggest that $25(\mathrm{OH}) \mathrm{D}_{3}$ and vitamin $\mathrm{D}$ binding protein may even be protective factors for thyroid cancer and vitamin $\mathrm{D}$ deficiency may be a risk factor for thyroid cancer.

\section{Related Hormones as Markers}

As we all know, the incidence of thyroid cancer has a very clear and obvious gender difference, which is easily reminiscent of the effects of sex-related hormones. However, the expression of estrogen receptors in thyroid tissue has many different and even some contradictory results. And because sex-related hormones, such as estradiol, are affected by numerous factors, they are not very sensitive and specific. Therefore, despite the fact that many studies have shown that there are multiple ERs in thyroid tissue, ${ }^{30,31}$ and played an important role in the pathophysiology of thyroid cancer, but it may not be suitable as a diagnostic marker.

There is another important hormone that cannot be avoided in the occurrence and development of thyroid cancer, which is thyroid stimulating hormone (TSH). As the main growth factor of thyroid cells, TSH is a regulator of thyroid function, and its importance as a predictor of thyroid cancer risk has been widely recognized. At 
present, TSH inhibition therapy is widely used in clinical patients after surgery to reduce thyroid cancer recurrence rate and improve patient survival. ${ }^{3}$ Although there is some evidence that TSH levels in patients with thyroid cancer will increase, the cutoff is difficult to determine, so based on TSH alone cannot make diagnosis. ${ }^{32}$ And about whether the TSH level rises in thyroid cancer patients before surgery is actually still controversial. In Huang's study, it was suggested that TSH levels showed opposite effects in different genders, and were also affected by various pathological subtypes of thyroid cancer. ${ }^{33}$

\section{Tumor-Related Markers}

As we all know, chronic inflammation is related to the occurrence of cancer, and calprotectin is involved in the occurrence and development of various inflammations. Tabur et al found that the concentration of calprotectin in the serum of PTC patients increased significantly, and then decreased significantly after total thyroidectomy, besides, the level was similar to that of healthy people. Therefore, perhaps an increase in calprotectin may be a potential marker for oxidative stress in thyroid cancer. But again, the increase in calprotectin can occur in many diseases, so its specificity is not satisfactory. ${ }^{34}$ Besides, Argyris summarized some searches and found that only malignant thyroid cancer which has aggressive characteristics would has increased calprotectin level. In addition to anaplastic thyroid carcinoma, it has no significant role in non-neoplastic thyroid tissue and well-differentiated thyroid tumors. ${ }^{35}$

And as an important proinflammatory factor, platelets have also been studied as a biomarker. It will be rather convenient if the platelets could be the marker, since the test of it is such regular. However, the results are totally different and contradictory. The study by Baldane et al in 2015 included 98 people shows the mean platelet volume (MPV) is higher in PTC patients, and gave a cut-off value as $7.81 \mathrm{fl}^{36} \mathrm{Yu}$ et al investigated 280 people in 2017 attained a complete opposite result with PTC patients have lower MPV and higher platelet distribution width (PDW). ${ }^{37}$ While in Dincel's study there's no significant difference in MPV, but PDW is lower and plateletcrit is higher in PTC patients. ${ }^{38}$

For patients with tumor diseases, intact tumor cells and cell-free nucleic acids (cfDNA/cfRNA and circulating miRNA) can all appear in peripheral blood. ${ }^{39}$ And given the tissue-specific expression pattern and stability of miRNAs, circulating miRNAs have become ideal biomarkers for many cancers. Among the most often mentioned and proven circulating substances in PTC patients are cfDNA, ${ }^{40,41} \operatorname{lnc}-\mathrm{RNAs}^{42}$ and various miRNAs. Among them, levels of miR-146, miR-221, miR-222 and let-7 were higher than those of the healthy control group ${ }^{43-48}$ and miR-222 and miR-146b can distinguish between PTC and benign nodules. What's more, high-mobility group box-1 has also been proposed to be a marker, ${ }^{49}$ and as Mardente studied, its interact with RAGE enhances the level of miR221/222 that in turn inhibits tumor suppressor gene PTEN. ${ }^{50}$

Midkine is a pleiotropic growth factor that is significantly expressed during embryogenesis and regulates cell growth, survival, migration, angiogenesis, and antiapoptotic activities, but usually has low expression levels in adulthood. Some studies have shown that midkine expression in PTC is strong, and it is related to the clinicopathological characteristics and metastasis of PTC. ${ }^{51,52}$ Meng ${ }^{53}$ and $\mathrm{Li}^{54}$ have successively confirmed midkine's potential as a marker of thyroid cancer. Moreover, Meng et al used $323.12 \mathrm{pg} / \mathrm{mL}$ as the cutoff value to distinguish benign and malignant thyroid nodules and obtained its sensitivity, specificity, and diagnostic accuracy rates of $75.70 \%, 75.00 \%$, and $75.31 \%$, respectively. But in fact, it has increased in more than 20 tumor diseases, so it needs to be assisted by other means.

Interleukin (IL) is a small protein signal molecule mainly synthesized by $\mathrm{T}$ cells, monocytes, macrophages and endothelial cells. It plays an important role in promoting communication between immune system cells, regulating transcription factors, controlling inflammation, differentiation, proliferation, and secreting antibodies etc. Various interleukins have been widely recognized as diagnostic, prognostic indicators and treatment methods. In a validation test by Martins et al, the levels of IL-6, IL8 , and IL-10 were higher in patients with thyroid nodules than in healthy people, but they could not distinguish between benign and malignant nodules. In addition to the sensitivity of IL-2 reaching $98 \%$, the others' sensitivity and specificity are not ideal. ${ }^{55}$

Matrix metalloproteinase (MMP) is a zinc-dependent endopeptidase, which has the function of digesting gelatin and a variety of collagens, and is involved in the development of many tumors. Shi et al affirmed its value as a diagnostic marker of thyroid cancer in their research. The serum MMP-2 concentration of PTC patients is greater than of healthy people, and its level can be reduced after surgery. In addition, it may also have significant effects in prognostic evaluation and treatment. ${ }^{56}$ Zhang et al also 
affirmed the role of MMP-2 in their research. At the same time, they also proposed that MMP-9 and TIMP-1, and TIMP-2 may have analogous effects, and the imbalance between MMP and TIMP may cause tumor progression. ${ }^{57}$

Vascular adhesion protein-1 (VAP-1) is an endothelial cell adhesion molecule that is involved in the process of leukocyte rolling, adhesion and transfer to inflammatory sites. Hu et al measured serum levels of VAP-1 in patients with thyroid cancer and benign thyroid adenomas, and found that serum VAP-1 levels in the thyroid cancer group were significantly lower than those in the healthy control group and the benign nodule group. And it negatively correlated with serum thyroglobulin concentration in patients with thyroid cancer. The optimal cutoff value of VAP-1 for diagnosis of thyroid cancer was $456.6 \mathrm{ng} / \mathrm{mL}$, with a specificity of $77.4 \%$ and a sensitivity of $66.7 \%{ }^{58}$ Meanwhile, Baki's research in 2019 also confirmed its value as a potential marker. ${ }^{59}$

Galectin-3 is a protein that binds to $\beta$-galactosyl residues of cell surface glycoproteins. Many studies have shown that its expression levels are different in benign and malignant thyroid tissues. ${ }^{60-62}$ The study of Saussez in 2008 showed that the level of Galectin-3 in the serum of patients with thyroid disease is higher than that of healthy people. ${ }^{63}$ Yilmaz's research in 2015 affirmed the statistical value of the difference, but in view of the inadequacy of existing studies, it was considered that it can only be used as a method of auxiliary diagnosis. ${ }^{64}$

$\beta-2$ microglobulin is the light chain of human leukocyte antigen (HLA). Studies by Adil et al suggest that after excluding the deposition of $\beta$ - 2 microglobulin in the blood due to renal insufficiency, $\beta-2$ microglobulin elevated concentrations in serum can also be used as auxiliary diagnostic markers of thyroid cancer. ${ }^{65}$

CYFRA21-1 is a fragment of CK19, whose washout fluid shows great potential in diagnose the metastatic lymph nodes of DTC when combined with FNA. ${ }^{66}$ Though its concentration in serum presents good value in diagnosing head and neck cancer, including oral, oropharyngeal, hypopharyngeal, and laryngeal cancers, ${ }^{67}$ it do not perform well in discerning benign and malignant thyroid nodules. Early to 2008 and 2010, someone has claimed that its level has no manifested difference between benign and malignant thyroid nodules. But like Tg, it may help in predicting the prognosis. ${ }^{68}$ Besides, the higher level of it may indicate the poorly differentiated and anaplastic thyroid carcinoma, and worse prognosis of DTC.

\section{Discussion}

As an important endocrine organ of the human body, the thyroid gland plays an important role in the growth and development of the human body and normal physiological activities, and involves in various physiological processes. Because of this, the mechanism of the occurrence and development of thyroid disease seems to be particularly complicated. The incidence of thyroid disease is increasing year by year, and the largest part of it is PTC. Although with the increasingly standardized treatment of thyroid cancer patients, the 5-year survival rate of patients with thyroid cancer has reached $99.4 \%,{ }^{1}$ but there are also questions about overdiagnosis and overtreatment. We have to admit that surgery is the cornerstone of not only DTC but almost all kinds of thyroid cancers. However, due to the inaccurate diagnosis, we may do some unnecessary surgeries, which also means some unnecessary risks, like the injury of parathyroid or recurrent laryngeal nerve, which may cannot be prevented. ${ }^{69}$

Given that the blood test and ultrasound have become the regular examinations when patients come to hospital for thyroid disease. This article summarizes potential markers in the blood of patients with PTC, hoping to find a marker that can sensitively and specifically diagnose PTC without adding another examination for patients.

We have listed plenty of biomarkers in categories, but as we can see, most of them are general markers for many cancers. And unfortunately, even the substances related to thyroid function, except calcitonin for medullary thyroid cancer, have not shown enough value in diagnosis, for their undefinite cut-off value. But the markers which have definite changes, like miR-221, miR-222 or midkine etc., between malignant and benign nodules may can help in confirming the existence of cancer, although they may not have satisfactory specificity. But combining different kinds of marker as a panel may have some help. Though the changes on metabolic profiles and circulatory system nucleic acids are rather complicated, but in my opinion, they do show a hopeful prospect compared to other markers. We may have some hope to find a substance in them that can balance sensitivity and specificity to help diagnose thyroid cancer in the future.

The biomarkers can discern the malignant nodules with benign ones can help us avoid the unnecessary surgeries. What's more, if they can predict the aggressiveness of the cancer, it may also help to decide the surgical method, for 
the extent of lymph node clearance is controversy for years, especially about the preventive clearance. For some poorly differentiated cancer or ATC, some paper proposed the combined treatment might improve the survival, but is better in early stage. ${ }^{70}$ So it is quite important to find a biomarker for ATC, like CYFRA21-1 and calprotectin might have the potential, in order to make the early diagnosis.

In summary, for more than 40 years, people have made many efforts in the search for thyroid cancer markers, but have not yet found a substance that can accurately diagnose PTC. Most of the substances above correspond to multiple diseases at the same time, thus cannot rely on a single one to diagnose thyroid cancer, but perhaps combined with several models can better distinguish patients with thyroid cancer.

\section{Disclosure}

The authors report no conflicts of interest in this work.

\section{References}

1. Siegel RL, Miller KD, Jemal A. Cancer statistics, 2018. CA Cancer J Clin. 2018;68(1):7-30. doi:10.3322/caac.21442

2. Kim J, Gosnell JE, Roman SA. Geographic influences in the global rise of thyroid cancer. Nat Rev Endocrinol. 2020;16(1):17-29. doi:10.1038/s41574-019-0263-x

3. Haugen BR, Alexander EK, Bible KC, et al. 2015 American thyroid association management guidelines for adult patients with thyroid nodules and differentiated thyroid cancer: the American thyroid association guidelines task force on thyroid nodules and differentiated thyroid cancer. Thyroid. 2016;26(1):1-133. doi:10.1089/thy.2015.0020

4. Khatami F, Payab M, Sarvari M, et al. Oncometabolites as biomarkers in thyroid cancer: a systematic review. Cancer Manag Res. 2019;11:1829-1841. doi:10.2147/CMAR.S188661

5. Grogan RH, Mitmaker EJ, Clark OH. The evolution of biomarkers in thyroid cancer-from mass screening to a personalized biosignature. Cancers (Basel). 2010;2(2):885-912. doi:10.3390/cancers2020885

6. Melvin KEW, Miller HH, Tashjian AH. Early diagnosis of medullary carcinoma of the thyroid gland by means of calcitonin assay. $N$ Engl J Med. 1971;285(20):1115-1120.

7. Milhaud G, Calmette C, Taboulet J, Julienne A, Moukhtar MS. Letter: hypersecretion of calcitonin in neoplastic conditions. Lancet. 1974;1(7855):462-463. doi:10.1016/S0140-6736(74)92428-3

8. Alfarouk KO, Verduzco D, Rauch C, et al. Glycolysis, tumor metabolism, cancer growth and dissemination. A new pH-based etiopathogenic perspective and therapeutic approach to an old cancer question. Oncoscience. 2014;1(12):777-802. doi:10.18632/oncoscience.109

9. Wojtowicz W, Zabek A, Deja S, et al. Serum and urine (1)H NMR-based metabolomics in the diagnosis of selected thyroid diseases. Sci Rep. 2017;7(1):9108. doi:10.1038/s41598-017-09203-3

10. Farrokhi Yekta R, Rezaei Tavirani M, Arefi Oskouie A, MohajeriTehrani MR, Soroush AR, Akbarzadeh Baghban A. Serum-based metabolic alterations in patients with papillary thyroid carcinoma unveiled by non-targeted 1H-NMR metabolomics approach. Iran J Basic Med Sci. 2018;21(11):1140-1147. doi:10.22038/JJBMS.2018.30375.7323

11. Huang FQ, Li J, Jiang L, et al. Serum-plasma matched metabolomics for comprehensive characterization of benign thyroid nodule and papillary thyroid carcinoma. Int J Cancer. 2019;144(4):868-876. doi:10.1002/ijc. 31925
12. Wojakowska A, Cole LM, Chekan M, et al. Discrimination of papillary thyroid cancer from non-cancerous thyroid tissue based on lipid profiling by mass spectrometry imaging. Endokrynol Pol. 2018;69 (1):2-8. doi:10.5603/EP.a2018.0003

13. Guo S, Qiu L, Wang Y, et al. Tissue imaging and serum lipidomic profiling for screening potential biomarkers of thyroid tumors by matrix-assisted laser desorption/ionization-fourier transform ion cyclotron resonance mass spectrometry. Anal Bioanal Chem. 2014;406(18):4357-4370. doi:10.1007/s00216-014-7846-0

14. Lee GB, Lee JC, Moon MH. Plasma lipid profile comparison of five different cancers by nanoflow ultrahigh performance liquid chromatography-tandem mass spectrometry. Anal Chim Acta. 2019;1063:117-126. doi:10.1016/j.aca.2019.02.021

15. Lu ZL, Chen YJ, Jing XY, Wang NN, Zhang T, Hu CJ. Detection and identification of serum peptides biomarker in papillary thyroid cancer. Med Sci Monit. 2018;24:1581-1587. doi:10.12659/MSM.907768

16. Evans C, Tennant S, Perros P. Serum thyroglobulin in the monitoring of differentiated thyroid cancer. Scand J Clin Lab Invest Suppl. 2016;245:S119-S123. doi:10.1080/00365513.2016.1210339

17. Indrasena BS. Use of thyroglobulin as a tumour marker. World J Biol Chem. 2017;8(1):81-85. doi:10.4331/wjbc.v8.i1.81

18. Kim H, Kim YN, Kim HI, et al. Preoperative serum thyroglobulin predicts initial distant metastasis in patients with differentiated thyroid cancer. Sci Rep. 2017;7(1):16955. doi:10.1038/s41598-01717176-6

19. Kim H, Park SY, Choe J-H, et al. Preoperative serum thyroglobulin and its correlation with the burden and extent of differentiated thyroid cancer. Cancers (Basel). 2020;12(3):625. doi:10.3390/cancers 12030625

20. Unluhizarci K, Akgun H, Oz B, Karaca Z, Tanriverdi F, Kelestimur F. Patients with papillary thyroid carcinoma associated with high stimulated serum calcitonin levels. Endocrinol Diabetes Metab Case Rep. 2017;2017.

21. Toledo SP, Lourenco DM Jr, Santos MA, Tavares MR, Toledo RA, Correia-Deur JE. Hypercalcitoninemia is not pathognomonic of medullary thyroid carcinoma. Clinics (Sao Paulo). 2009;64 (7):699-706. doi:10.1590/S1807-59322009000700015

22. Shen F, Cai WS, Li JL, Feng Z, Cao J, Xu B. The association between serum levels of selenium, copper, and magnesium with thyroid cancer: a meta-analysis. Biol Trace Elem Res. 2015;167 (2):225-235. doi:10.1007/s12011-015-0304-9

23. de Oliveira Maia M, Batista BAM, Sousa MP, de Souza LM, Maia CSC. Selenium and thyroid cancer: a systematic review. Nutr Cancer. 2019;1-9. doi:10.1080/01635581.2019.1679194

24. Rayman MP. Selenium and human health. Lancet. 2012;379 (9822):1256-1268. doi:10.1016/S0140-6736(11)61452-9

25. Choi YM, Kim WG, Kim TY, et al. Serum vitamin D3 levels are not associated with thyroid cancer prevalence in euthyroid subjects without autoimmune thyroid disease. Korean J Intern Med. 2017;32 (1):102-108. doi:10.3904/kjim.2015.090

26. Danilovic DL, Ferraz-de-Souza B, Fabri AW, et al. 25hydroxyvitamin D and TSH as risk factors or prognostic markers in thyroid carcinoma. PLoS One. 2016;11(10):e0164550. doi:10.1371/ journal.pone. 0164550

27. Zhang T, Zhang $\mathrm{H}$, $\mathrm{He} \mathrm{L}$, et al. Potential use of 1-25dihydroxyvitamin $\mathrm{D}$ in the diagnosis and treatment of papillary thyroid cancer. Med Sci Monit. 2018;24:1614-1623.

28. Hu MJ, Niu QS, Wu HB, et al. Association of thyroid cancer risk with plasma 25 -hydroxyvitamin D and vitamin D binding protein: a case-control study in China. $J$ Endocrinol Invest. 2019:1-10.

29. Zhao J, Wang H, Zhang Z, et al. Vitamin D deficiency as a risk factor for thyroid cancer: a meta-analysis of case-control studies. Nutrition. 2019;57:5-11. doi:10.1016/j.nut.2018.04.015

30. Rajoria S, Suriano R, George AL, et al. Estrogen activity as a preventive and therapeutic target in thyroid cancer. Biomed Pharmacother. 2012;66(2):151-158. doi:10.1016/j.biopha.2011.11. 010 
31. Derwahl M, Nicula D. Estrogen and its role in thyroid cancer. Endocr Relat Cancer. 2014;21(5):T273-T283. doi:10.1530/ERC-14-0053

32. Jiaojiao Zheng CL, Weihui L, Wang C, Zhilong A. Quantitative assessment of preoperative serum thyrotropin level and thyroid cancer. Oncotarget. 2016;7(23):34918-34929. doi:10.18632/oncotarget. 9201

33. Huang H, Rusiecki J, Zhao N, et al. Thyroid-stimulating hormone, thyroid hormones, and risk of papillary thyroid cancer: a nested case-control study. Cancer Epidemiol Biomarkers Prev. 2017;26 (8):1209-1218. doi:10.1158/1055-9965.EPI-16-0845

34. Tabur S, Korkmaz H, Ozkaya M, et al. Serum calprotectin: a new potential biomarker for thyroid papillary carcinoma. Tumour Biol. 2015;36(10):7549-7556. doi:10.1007/s13277-015-3468-1

35. Argyris PP, Slama ZM, Ross KF, Khammanivong A, Herzberg MC. Calprotectin and the initiation and progression of head and neck cancer. J Dent Res. 2018;97(6):674-682. doi:10.1177/00220345187 56330

36. Baldane S, Ipekci SH, Sozen M, Kebapcilar L. Mean platelet volume could be a possible biomarker for papillary thyroid carcinomas. Asian Pac J Cancer Prev. 2015;16(7):2671-2674. doi:10.7314/APJCP.20 15.16.7.2671

37. Yu YJ, Li N, Yun ZY, et al. Preoperative mean platelet volume and platelet distribution associated with thyroid cancer. Neoplasma. 2017;64(4):594-598. doi:10.4149/neo_2017_414

38. Dincel O, Bayraktar C. Evaluation of platelet indices as a useful marker in papillary thyroid carcinoma. Bratisl Lek Listy. 2017;118 (3):153-155. doi:10.4149/BLL_2017_030

39. Cai X, Janku F, Zhan Q, Fan J-B. Accessing genetic information with liquid biopsies. Trends Genet. 2015;31(10):564-575. doi:10.1016/j. tig.2015.06.001

40. Caglar O, Cilgin B, Eroglu M, Cayir A. Evaluation of circulating cell free DNA in plasma as a biomarker of different thyroid diseases. Braz J Otorhinolaryngol. 2019.

41. Perdas E, Stawski R, Kaczka K, Nowak D, Zubrzycka M. Altered levels of circulating nuclear and mitochondrial DNA in patients with papillary thyroid cancer. Sci Rep. 2019;9(1):14438. doi:10.1038/ s41598-019-51000-7

42. Sedaghati M, Kebebew E. Long noncoding RNAs in thyroid cancer. Curr Opin Endocrinol Diabetes Obes. 2019;26(5):275-281. doi:10. 1097/MED.0000000000000497

43. Yu S, Liu Y, Wang J, et al. Circulating microRNA profiles as potential biomarkers for diagnosis of papillary thyroid carcinoma. $J$ Clin Endocrinol Metab. 2012;97(6):2084-2092. doi:10.1210/ jc.2011-3059

44. Lee JC, Zhao JT, Clifton-Bligh RJ, et al. MicroRNA-222 and microRNA-146b are tissue and circulating biomarkers of recurrent papillary thyroid cancer. Cancer. 2013;119(24):4358-4365. doi: $10.1002 /$ cncr.28254

45. Graham ME, Hart RD, Douglas S, et al. Serum microRNA profiling to distinguish papillary thyroid cancer from benign thyroid masses. J Otolaryngol Head Neck Surg. 2015;44(1):33. doi:10.1186/s40463015-0083-5

46. Rosignolo F, Sponziello M, Giacomelli L, et al. Identification of thyroid-associated serum microRNA profiles and their potential use in thyroid cancer follow-up. $J$ Endocr Soc. 2017;1(1):3-13. doi:10.1210/js.2016-1032

47. Rezaei M, Khamaneh AM, Zarghami N, Vosoughi A, Hashemzadeh S. Evaluating pre- and post-operation plasma miRNAs of papillary thyroid carcinoma (PTC) patients in comparison to benign nodules. BMC Cancer. 2019;19(1):690. doi:10.1186/ s12885-019-5849-0

48. Zhang A, Wang C, Lu H, et al. Altered serum MicroRNA profile may serve as an auxiliary tool for discriminating aggressive thyroid carcinoma from nonaggressive thyroid cancer and benign thyroid nodules. Dis Markers. 2019;2019:3717683. doi:10.1155/2019/ 3717683
49. Chuanyou C, Quansheng W, Yuzhong Q, Jianing L, Bo L, Bin L. Clinical value of combination detection of HMGB-1 and TSGF for diagnosis of thyroid cancer. Chin J Gen Surg. 2012;21(05):536-539.

50. Mardente S, Mari E, Massimi I, et al. HMGB1-induced cross talk between PTEN and miRs 221/222 in thyroid cancer. Biomed Res Int. 2015;2015:512027. doi:10.1155/2015/512027

51. Choi YW, Kim YH, Lee J, Soh EY, Park TJ, Kim JH. Strong immunoexpression of midkine is associated with multiple lymph node metastases in BRAFV600E papillary thyroid carcinoma. Hum Pathol. 2015;46(10):1557-1565. doi:10.1016/j.humpath.2015.06.018

52. Kuzu F, Arpaci D, Unal M, et al. Midkine: a novel biomarker to predict malignancy in patients with nodular thyroid disease. Int J Endocrinol. 2016;2016:6035024. doi:10.1155/2016/6035024

53. Meng Z, Tan J, Zhang G, et al. Evaluation of serum midkine as a biomarker in differentiated thyroid cancer. Life Sci. 2015;130:18-24. doi:10.1016/j.lfs.2015.02.028

54. Li N, Zhang C, Meng Z, et al. Changes of serum midkine as a dynamic prognostic factor to monitor disease status in papillary thyroid cancer. Medicine (Baltimore). 2018;97(36):e12242. doi:10.10 97/MD.0000000000012242

55. Martins MB, Marcello MA, Batista FA, et al. Serum interleukin measurement may help identify thyroid cancer patients with active disease. Clin Biochem. 2018;52:1-7. doi:10.1016/j.clinbiochem. 2017.10.003

56. Shi $\mathrm{Y}, \mathrm{Su} \mathrm{C}, \mathrm{Hu} \mathrm{H}$, et al. Serum MMP-2 as a potential predictive marker for papillary thyroid carcinoma. PLoS One. 2018;13(6): e0198896. doi:10.1371/journal.pone.0198896

57. Zhang WJ, Song B, Yang T. MMP-2, MMP-9, TIMP-1, and TIMP-2 in the peripheral blood of patients with differentiated thyroid carcinoma. Cancer Manag Res. 2019;11:10675-10681. doi:10.2147/ CMAR.S233776

58. Hu Z, Zhao P, Zhang K, Zang L, Liao H, Ma W. Evaluation of serum vascular adhesion protein-1 as a potential biomarker in thyroid cancer. Int $J$ Endocrinol. 2016;2016:6312529. doi:10.1155/2016/ 6312529

59. Baki A, Tosun İ, Yıldız M. Can VAP-1 protein be used as a biomarker in thyroid cancer? Istanb Med J. 2019;20(6):335-340. doi:10.4274/imj.galenos.2019.26817

60. Htwe TT, Karim N, Wong J, Jahanfar S, Mansur MA. Differential expression of galectin-3 in advancing thyroid cancer cells: a clue toward understanding tumour progression and metastasis. Singapore Med J. 2010;51(11):856-859.

61. Saggiorato E, Cappia S, De Giuli P, et al. Galectin-3 as a presurgical immunocytodiagnostic marker of minimally invasive follicular thyroid carcinoma. J Clin Endocrinol Metab. 2001;86(11):5152-5158. doi: $10.1210 /$ jcem. 86.11 .8043

62. Arcolia V, Journe F, Wattier A, et al. Galectin-1 is a diagnostic marker involved in thyroid cancer progression. Int $J$ Oncol. 2017;51(3):760-770. doi:10.3892/ijo.2017.4065

63. Sven Saussez DG, Chantrain G, Pattou F, et al. Serum galectin-1 and galectin-3 levels in benign and malignant nodular thyroid disease. Thyroid. 2008;18(7):705-712

64. Yilmaz E, Karsidag T, Tatar C, Tuzun S. Serum galectin-3: diagnostic value for papillary thyroid carcinoma. Ulus Cerrahi Derg. 2015;31 (4):192-196. doi:10.5152/UCD.2015.2928

65. Adil A, Mohammed A, Abdulhafidh A. Beta-2-microglobulin as a marker in patients with thyroid cancer. Iraqi Acad Sci J. 2019;18 (1):18-22.

66. Lee J, Park HL, Jeong C-W, et al. CYFRA 21-1 in lymph node fine needle aspiration washout improves diagnostic accuracy for metastatic lymph nodes of differentiated thyroid cancer. Cancers (Basel). 2019;11(4):487. doi:10.3390/cancers 11040487

67. Liu L, Xie W, Xue P, Wei Z, Liang X, Chen N. Diagnostic accuracy and prognostic applications of CYFRA 21-1 in head and neck cancer: a systematic review and meta-analysis. PLoS One. 2019;14(5): e0216561. doi:10.1371/journal.pone.0216561 
68. Giovanella L, Imperiali M, Trimboli P. Role of serum cytokeratin 19 fragment (Cyfra 21.1) as a prognostic biomarker in patients with differentiated thyroid cancer. Sci Rep. 2017;7(1):7359. doi:10.1038/ s41598-017-07915-0

69. Gambardella C, Polistena A, Sanguinetti A, et al. Unintentional recurrent laryngeal nerve injuries following thyroidectomy: is it the surgeon who pays the bill? Int J Surg. 2017;41(Suppl 1):S55-S59. doi:10.1016/j.ijsu.2017.01.112
70. Conzo G, Polistena A, Calo PG, et al. Efficacy of combined treatment for anaplastic thyroid carcinoma: results of a multinstitutional retrospective analysis. Int J Surg. 2014;12(Suppl 1):S178-S182. doi:10.10 16/j.ijsu.2014.05.015

\section{Publish your work in this journal}

Cancer Management and Research is an international, peer-reviewed open access journal focusing on cancer research and the optimal use of preventative and integrated treatment interventions to achieve improved outcomes, enhanced survival and quality of life for the cancer patient.
The manuscript management system is completely online and includes a very quick and fair peer-review system, which is all easy to use. Visit http://www.dovepress.com/testimonials.php to read real quotes from published authors. 